\title{
Proposed Improvement of Forecasting Using Time Series Forecasting of Fast Moving Consumer Goods
}

\author{
Tasya Regina ${ }^{1 *}$, Panca Jodiawan ${ }^{2}$ \\ ${ }^{1}$ Department of Industrial Engineering, Universitas Bunda Mulia, Jl. Lodan Raya No. 2 Ancol, North Jakarta \\ 14430, Indonesia \\ ${ }^{2}$ Department of Industrial Management, National Taiwan University of Science and Technology, Keelung Rd \\ No. 43, Section 4, Da'an District, Taipei City 106, Taiwan
}

Received: 3 December, 2020 / Accepted: 8 February, 2021

\begin{abstract}
The company discussed in this paper is a national distributor firm that distributes FMCG products. The PPIC division in the company is responsible for forecasting the demand using the combination of the moving average method and intuition according to the interest of the company. However, the PPIC staff never measures the accuracy of their forecasting method. This research paper aims to evaluate the forecasting methods used to predict the demands of 12 classes of A SKU. Four-time series forecasting methods are particularly implemented, i.e., ARIMA, moving average (MA), double exponential smoothing (DES), and linear regression (RL). Forecasting using the ARIMA method is carried out by considering the stationarity of the average and variance of the historical data points. Forecasting using DES is carried out by using the optimal alpha and gamma values of the ARIMA method. The results show that the performance of each forecasting method varies, depending on which demands of class A SKU are predicted. Based on these results, the current forecasting method utilized by the company should be improved using the time series forecasting methods leading to the smallest error values for each class of A SKU.
\end{abstract}

Keyword:FMCG, Moving average, Minitab, ARIMA, Double exponential smoothing, Linear regression

\section{INTRODUCTION}

Fast-moving consumer goods (FMCG) are a part of the non-oil and gas industry. FMCG consists of daily needs with fast movements, such as food, clothing, and household needs. Although FMCG consumption has decreased due to changes in people's consumption priorities, FMCG is an essential need of every household thus its consumption will never stop.

Apart from being affected by priority changes in people's consumption, FMCG consumption is also affected by the flow of the supply chain. When each stage of the supply chain runs smoother, the consumer interest in buying these goods will also increase (Manders, Caniëls, \& Th, 2016). In this case, distribution activities are crucial to ensure a smooth supply chain. The distributor is a company that provides distribution services to manufacturing or retail companies. There is a difference in the distributor's role and it depends on the supply chain structure of each country (Chopra \& Meindl, 2016). The retail conditions in developed countries tend to be consolidated as a whole with the purchase of FMCG products in large quantities, while retail in developing countries has limitations in the storage systems so that FMCG products are purchased in small volumes (Chopra and Meindl, 2016). This can be reflected in the high contribution of transportation and savings to GDP in 2019, which is around 5.6\% (Hirschmann, 2020). Before COVID-19 pandemic, the development of domestic business is currently in a state of rapid growth (Tannady, Nurprihatin and Hartono, 2018)

The company discussed in this paper is a national distribution company engaged in the distribution of FMCG products from the $P \& G$ manufacturing company. The company encountered various challenges, such as a large amount of Stock Keeping Units $( \pm 1,900$ ID SKUs), fluctuations in SKU changes (upsize or downsize), managerial related issues, and limited company budgets. The Production Planning and Inventory Control (PPIC) division is a division at the company that has a responsibility in regulating the number of SKUs

*email: tasyaregina441@gmail.com 
that must be ordered and stored according to the predicted number of demand. For predicting the number of SKU demand, PPIC division employees currently use the combination between the Moving Average method and intuition based on current managerial interests. Furthermore, the accuracy of the implemented forecasting method is not measured. Consequently, it leads to potential lost sales and inventory issues at the beginning of each month. Therefore, any unnecessary inventory should be minimized if cannot be eliminated (Tannady et $a l ., 2019)$. The accumulation of the inventory can lead to a decrease in the quality (Christian et al., 2021). A previous study discussed the transportation of the inventory under the developed vehicle routing modeling (Nurprihatin and Lestari, 2020). Another research pointed out the distribution routes that are directly related to transportation costs (F. Nurprihatin et al., 2019) and solved using the heuristics method (Filscha Nurprihatin et al., 2019).

Based on the classification results carried out by the PPIC division, there are 12 SKUs included in class A items out of a total of 437 SKUs that are still active from January 2018 to the end of July 2020. Figure 1 presents one of the error measurements, i.e., the calculation of the mean absolute percentage error (MAPE), for 12 active SKUs of class $A$ based on the current forecasting method and actual demand data from companies. It can be seen that the current forecasting method produces an average error of $90.24 \%$. Therefore, an improvement in the forecasting method is crucial so that the demand prediction can be more accurate and closer to the actual conditions.

This study hence aims to evaluate several existing forecasting methods and perform a comparison between these forecasting methods with the ones currently used by the company. The comparison is conducted by comparing two types the forecasting accuracy measurements, i.e., Mean Absolute Deviation (MAD) and Mean Absolute Percentage Error (MAPE), obtained from four-time series forecasting methods, i.e., autoregressive integrated moving average (ARIMA), moving average (MA), double exponential smoothing (DES), and linear regression (RL). The results lead to an appropriate forecasting method for each SKU.

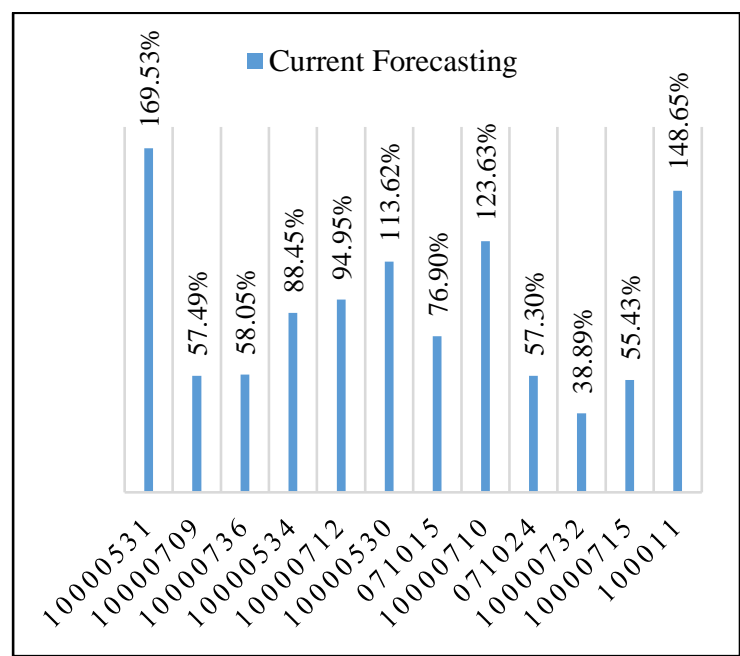

Figure 1. Error Measurement

(Source: Reseacher, 2020)

\section{METHODOLOGY}

\subsection{Research Objects}

This research analyses the object in the form of all classes of A SKU in the company. The collected datasets are the SKU classification data carried out by the company and the weekly demands from January 2018 to July 2020.

\subsection{Research Flow Chart}

The steps of this research start with collecting data. The collected data consists of SKU classification data from companies, weekly demands from January 2018 to July 2020, the forecasting demands of the company's PPIC division, and interviews with experts to determine the condition of the company. Afterward, four forecasting methods, i.e., ARIMA, MA, DES, and RL, are implemented using MiniTab 18 software. The results of the optimal ARIMA model will be used to determine the alpha value (level) and gamma values (trend) for the forecasting using the DES method. Furthermore, the MAD, MAPE, and MSE values are calculated as measurement indicators for each forecasting method, including the forecasting methods currently used by the company. These measurement indicators serve as a basis to determine the most appropriate forecasting method to predict the demand of each SKU. 


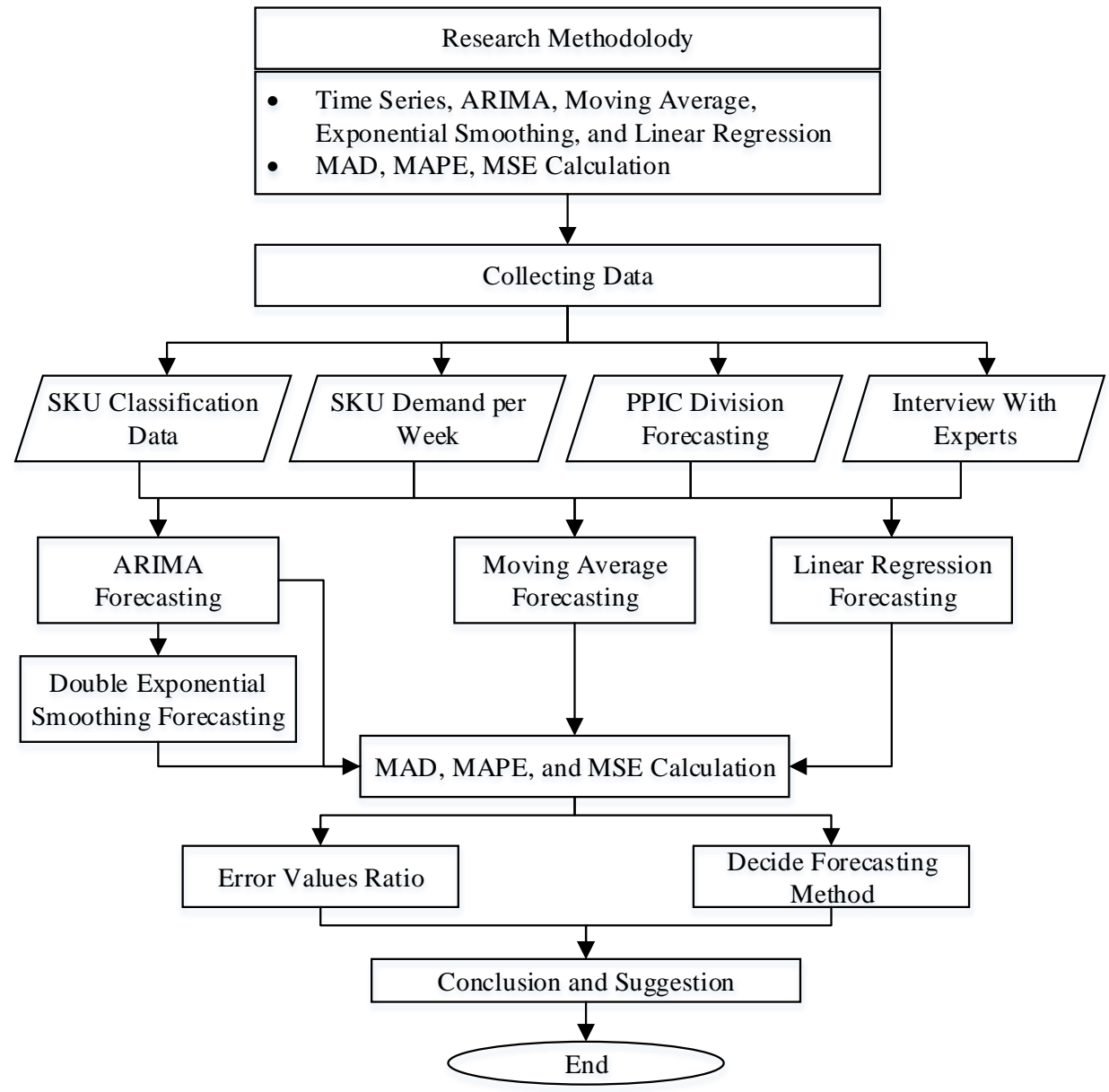

(Source: Researcher, 2020)

Figure 2. Flow Chart Research

The object of this research is class A SKU at the company. The selection of class A SKU as the object of research is because class A SKU is the SKU with the highest percentage of overall demand so that it contributes $80 \%$ of the company's revenue. The data collection phase is done by collecting primary data through direct observation in the field. In addition, primary data was obtained by conducting direct interviews with the patient manager of the company. Figure 2 shows a sequence of the research flow chart in conducting this research.

\section{RESULTS AND DISCUSSION}

ARIMA, moving average, double exponential smoothing, and linear regression forecasting are carried out for class A SKUs that are active from January 2018 to June 2020 based on the classification results that have been carried out by the PPIC division. There are 12 SKUs that will be forecasted, namely codes 10000531, 10000709, 10000736, 10000534,
10000712, 10000530, 071015, 10000710, 071024, 10000732, 10000715, 100011.

\subsection{ARIMA Forecasting}

Forecasting using the ARIMA method is carried out using Minitab 18 software. The stages of ARIMA forecasting using MiniTab 18 are described as follows:

1. Perform the average stationary test on the data by observing the distribution of trends in the data. There are 12 class A SKUs that will be tested for stationary trends.

2. Perform the variance stationary test on the data with a box-cox plot. Data with a stationary variance will have a rounded value $=1$. If the variance of the data is not stationary, data transformation must be performed.

3. Observe the distribution of trends from the transformed data. If the average of the data transformation is still not stationary then we need to differencing the data. 
4. Perform autocorrelation function (ACF) and partial autocorrelation function (PACF) testing phases after conducting the differencing data. If five lines in the data distribution cross the limit of the initial lag, the data cannot be used.

5. If the data can be used, then trial and error are carried out for determining the ARIMA model. Selection of the optimal ARIMA model is based on several criteria: (1) the pvalue model which must be $\leq 0.05$ so that the model can be said to be significant, (2) the pvalue on the L-Jung Box must be $\geq 0.05$ so that the residue on the model can be said to be of insignificant value, and (3) the model has the smallest mean square error (MSE).

Table 1 explains the ARIMA model that was selected after processing the data according to the steps above.

\subsection{Moving Average Forecasting (MA)}

Forecasting using the MA method takes the average length of the data for the past 3 periods (Maricar, 2019). Figures 3 to 14 illustrate the plot between the actual demand and the fitted demand produced by the MA forecasting method.

Table 1. ARIMA Model

\begin{tabular}{cccc}
\hline SKU & ARIMA Model $(\mathbf{p}, \mathbf{d}, \mathbf{q})$ & MS & Details \\
\hline 10000531 & $4,1,2$ & $2,487,108,080$ & Can be used \\
10000709 & $3,0,4$ & $4,649,265,827$ & Can be used \\
10000736 & $2,1,3$ & $2,343,548,943$ & Can be used \\
10000534 & $2,1,3$ & $1,174,064,338$ & Can be used \\
10000712 & $2,1,3$ & $1,469,822,041$ & Can be used \\
10000530 & $2,1,3$ & $695,614,178$ & Can be used \\
71015 & $3,0,3$ & $320,301,684$ & Can be used \\
10000710 & $5,1,2$ & $241,715,389$ & Can be used \\
71024 & $2,0,1$ & $202,293,232$ & Can be used \\
10000732 & $1,1,2$ & $348,122,109$ & Can be used \\
10000715 & $2,1,0$ & $29,433,241$ & Can be used \\
100011 & $4,1,3$ & $6,854,426$ & Can be used \\
\hline
\end{tabular}

(Sources: Primary Data Processing, 2020) 


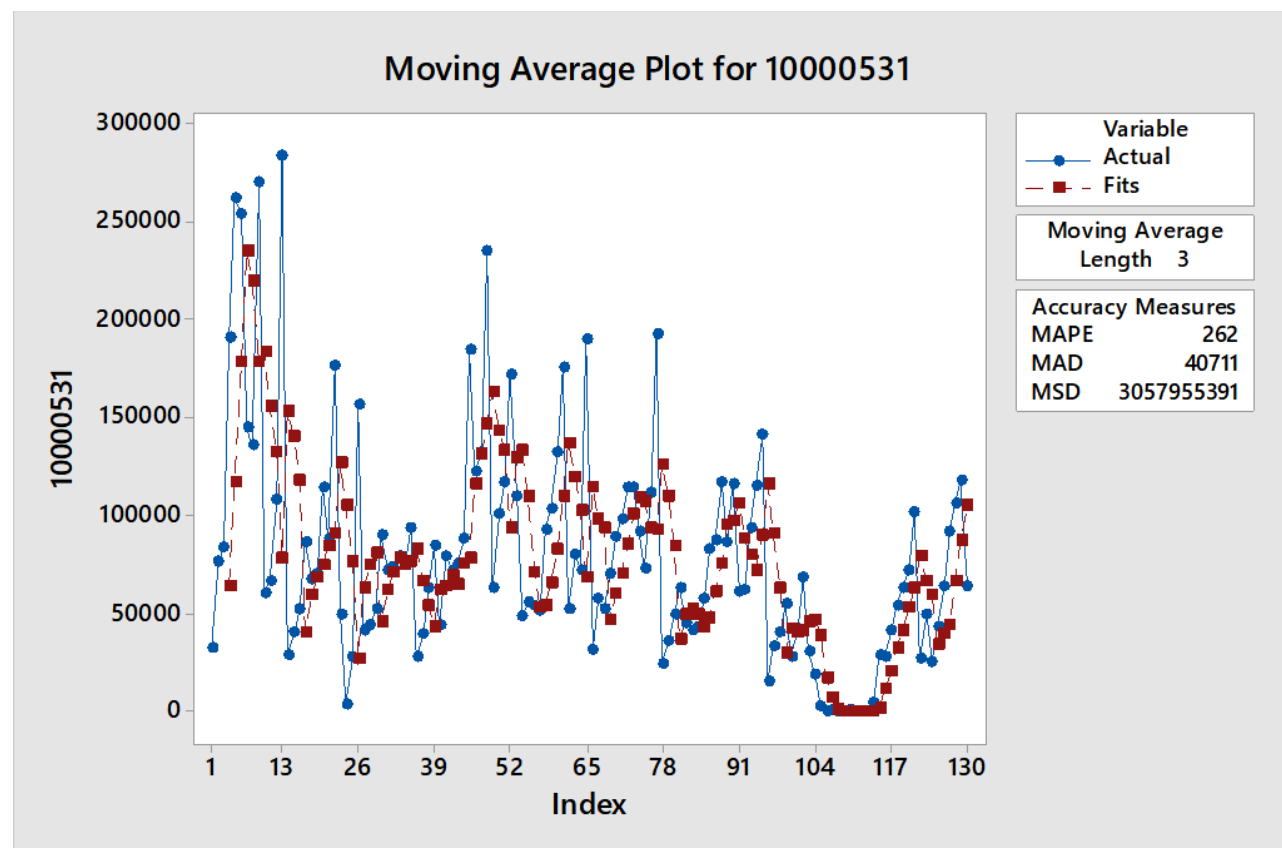

Figure 3. Moving Average SKU 10000531

(Source: Primary Data Processing, 2020)

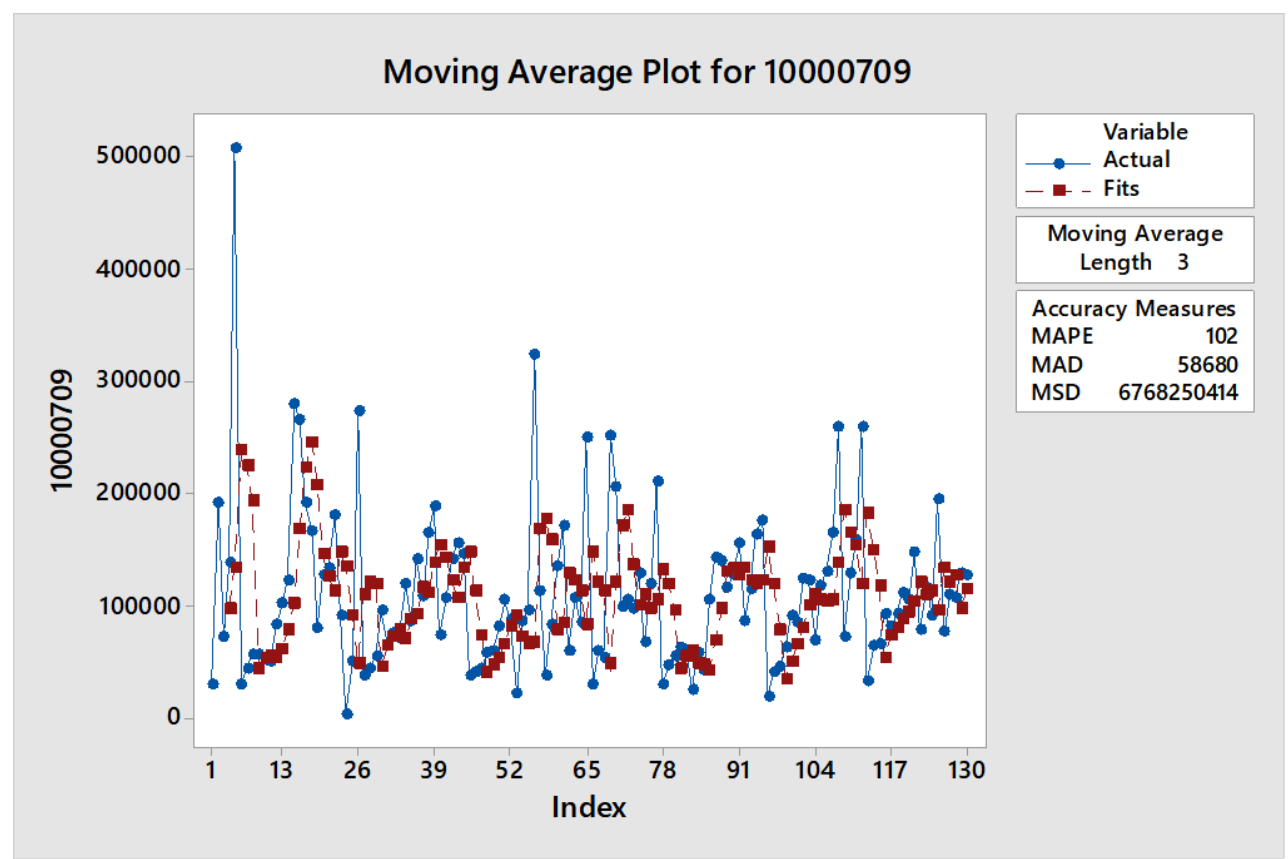

Figure 4. Moving Average SKU 10000709

(Source: Primary Data Processing, 2020) 


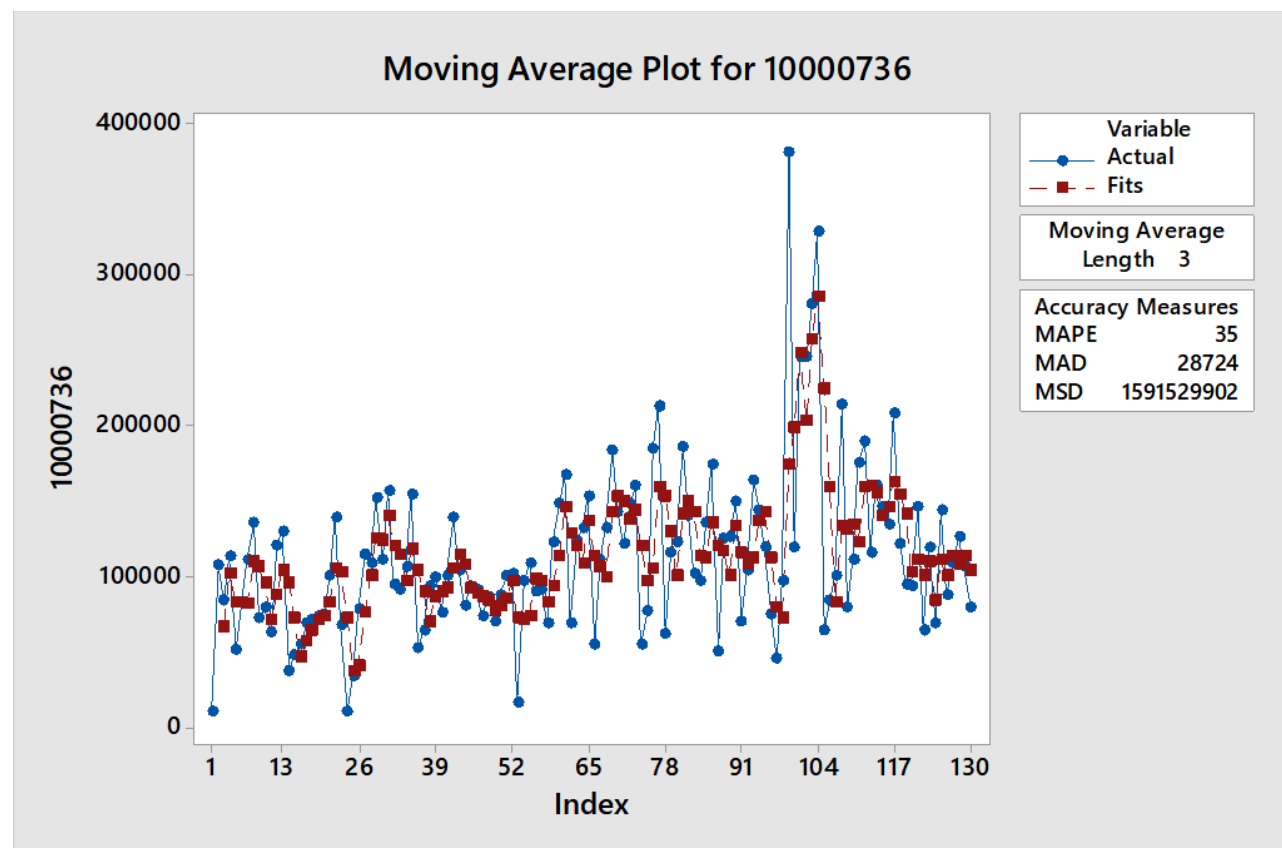

Figure 5. Moving Average SKU 10000736

(Source: Primary Data Processing, 2020)

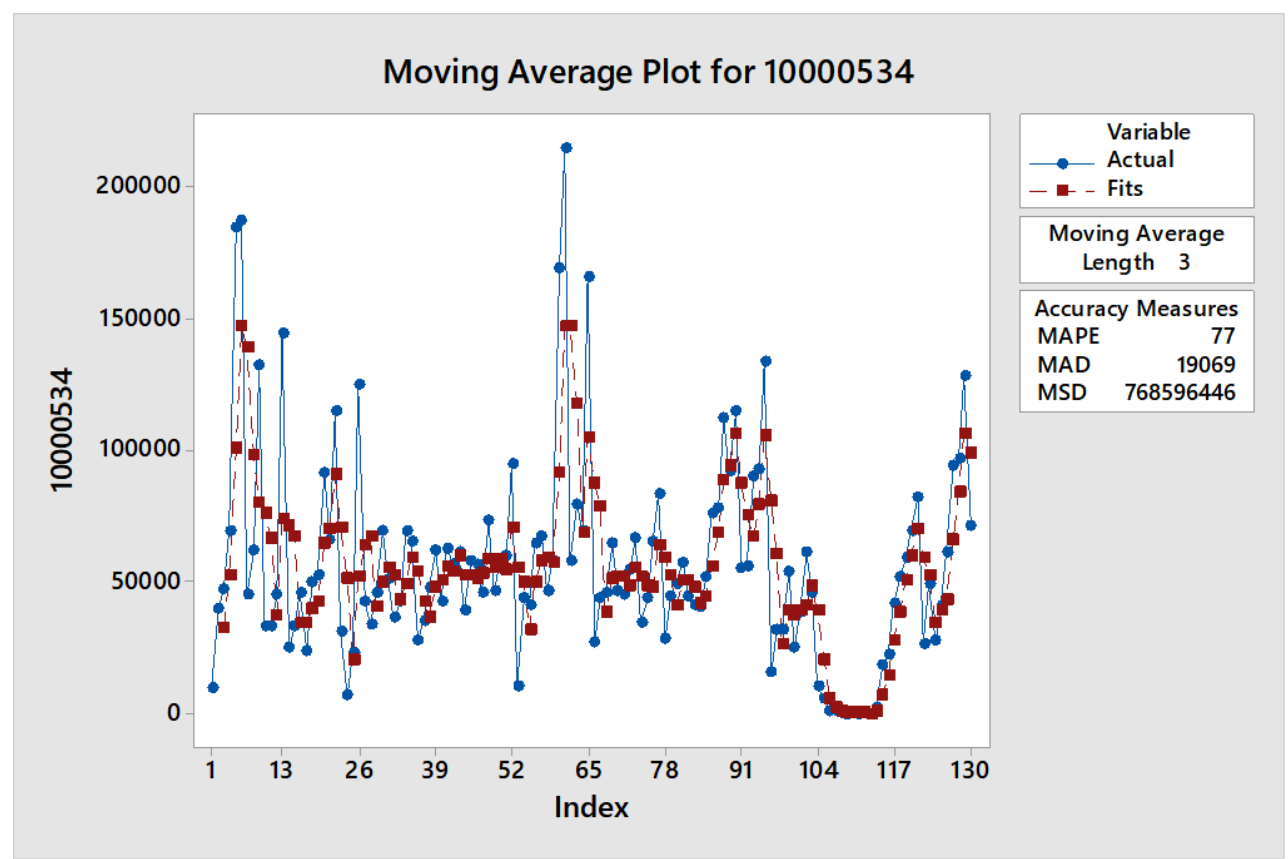

Figure 6. Moving Average SKU 10000534

(Source: Primary Data Processing, 2020) 


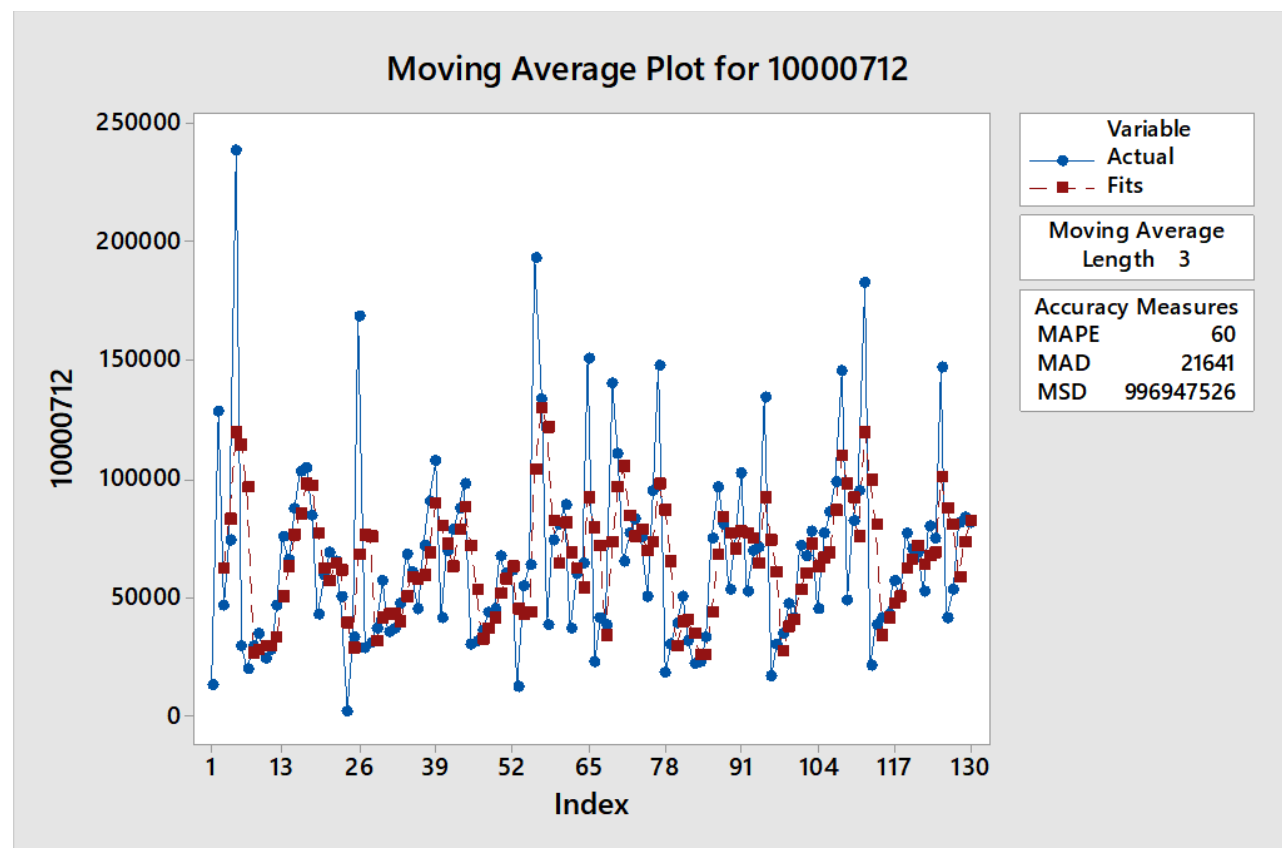

Figure 7. Moving Average SKU 10000712

(Source: Primary Data Processing, 2020)

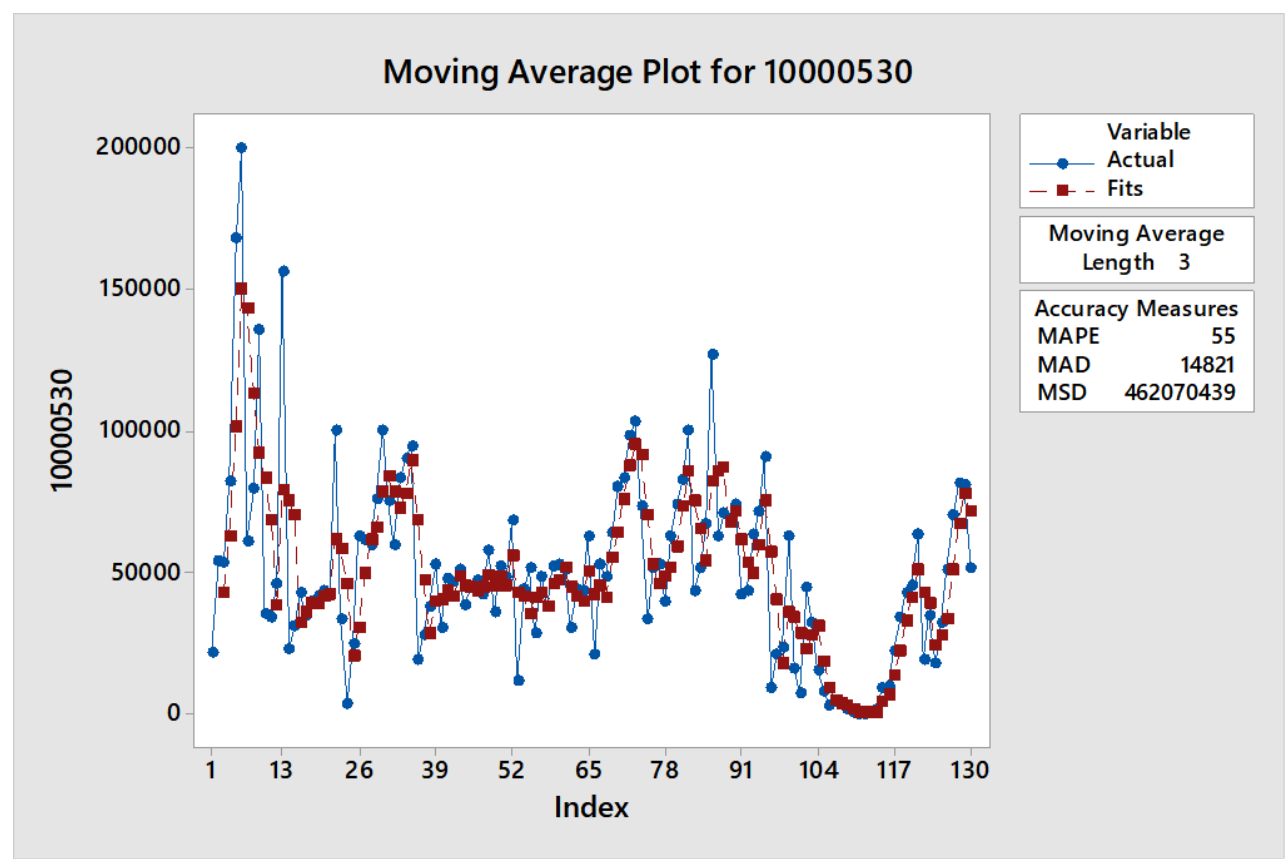

Figure 8. Moving Average SKU 10000530

(Source: Primary Data Processing, 2020) 


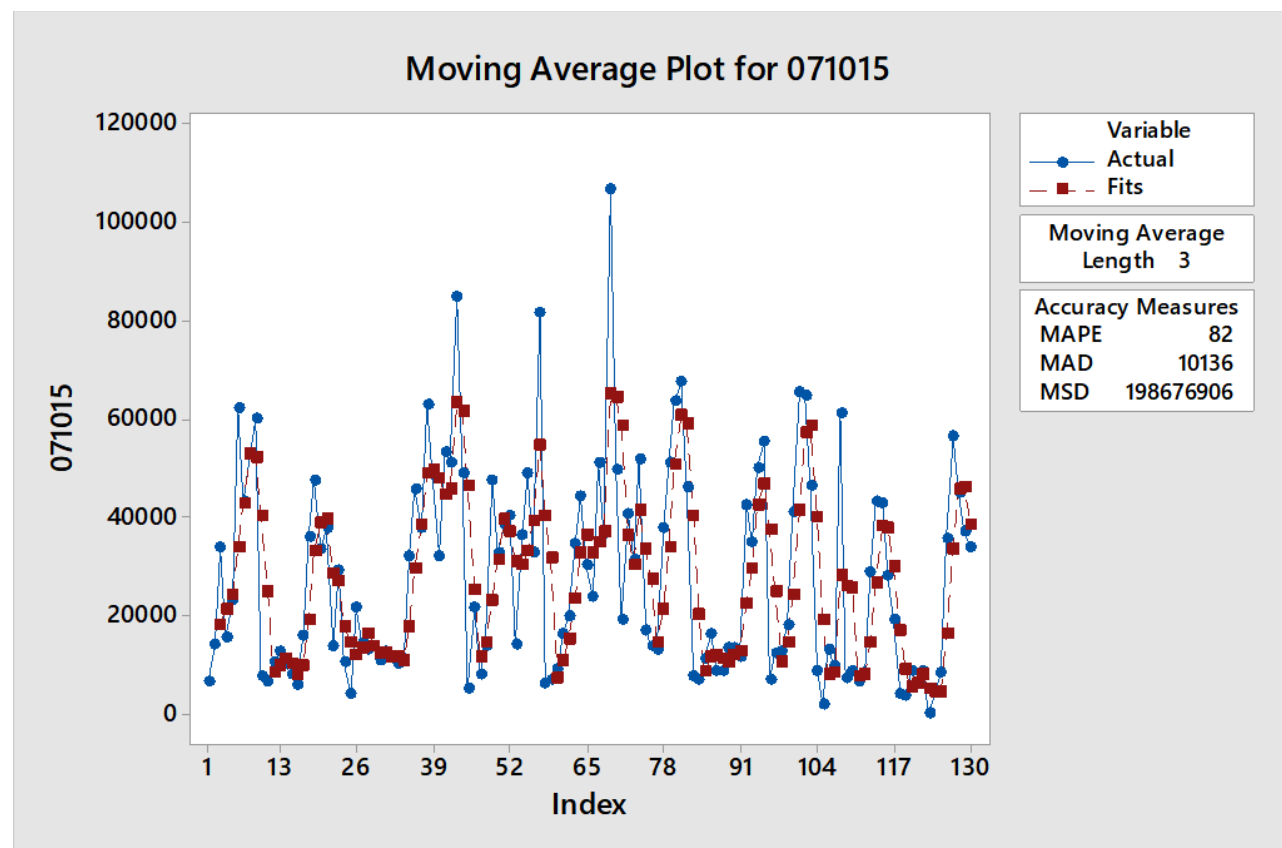

Figure 9. Moving Average SKU 071015

(Source: Primary Data Processing, 2020)

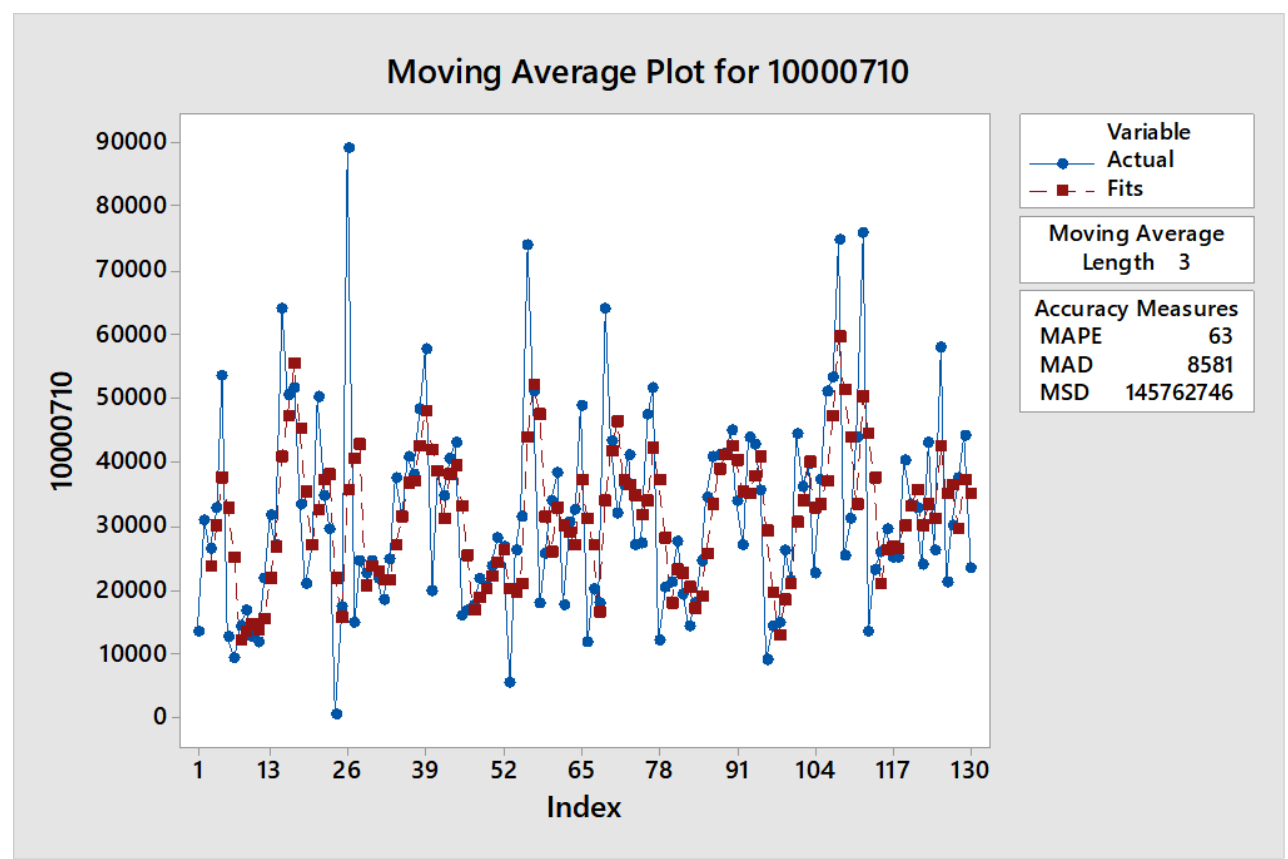

Figure 10. Moving Average SKU 10000710

(Source: Primary Data Processing, 2020) 


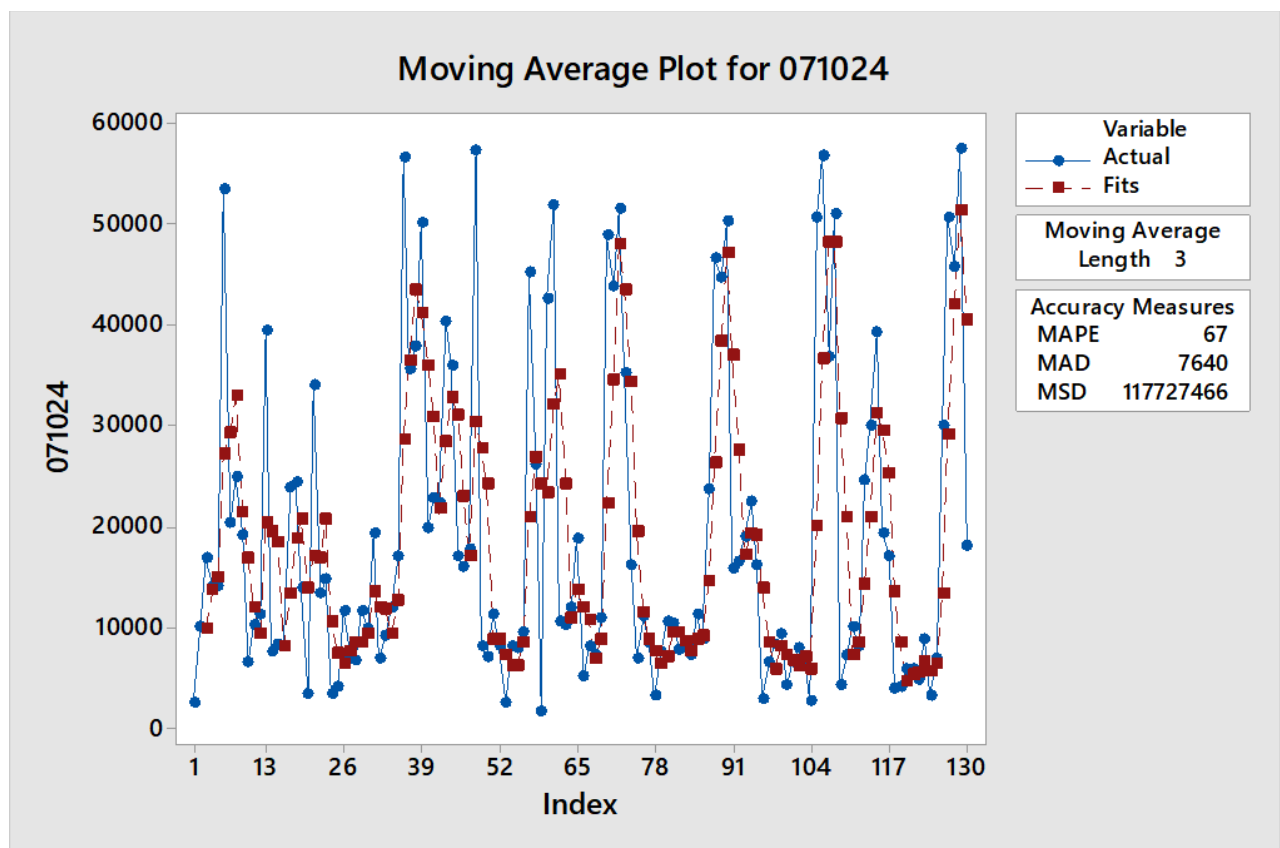

Figure 11. Moving Average SKU 071024

(Source: Primary Data Processing, 2020)

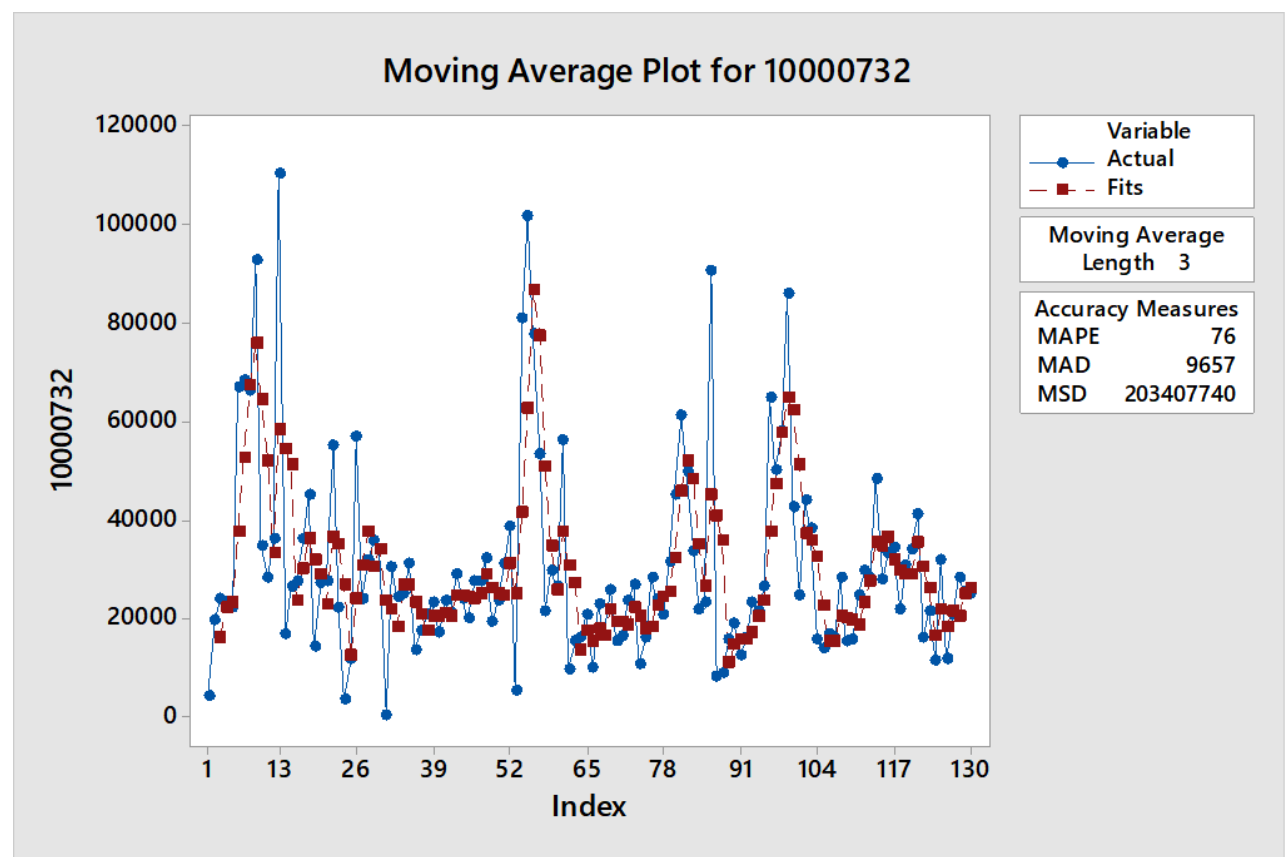

Figure 12. Moving Average SKU 10000732

(Source: Primary Data Processing, 2020) 


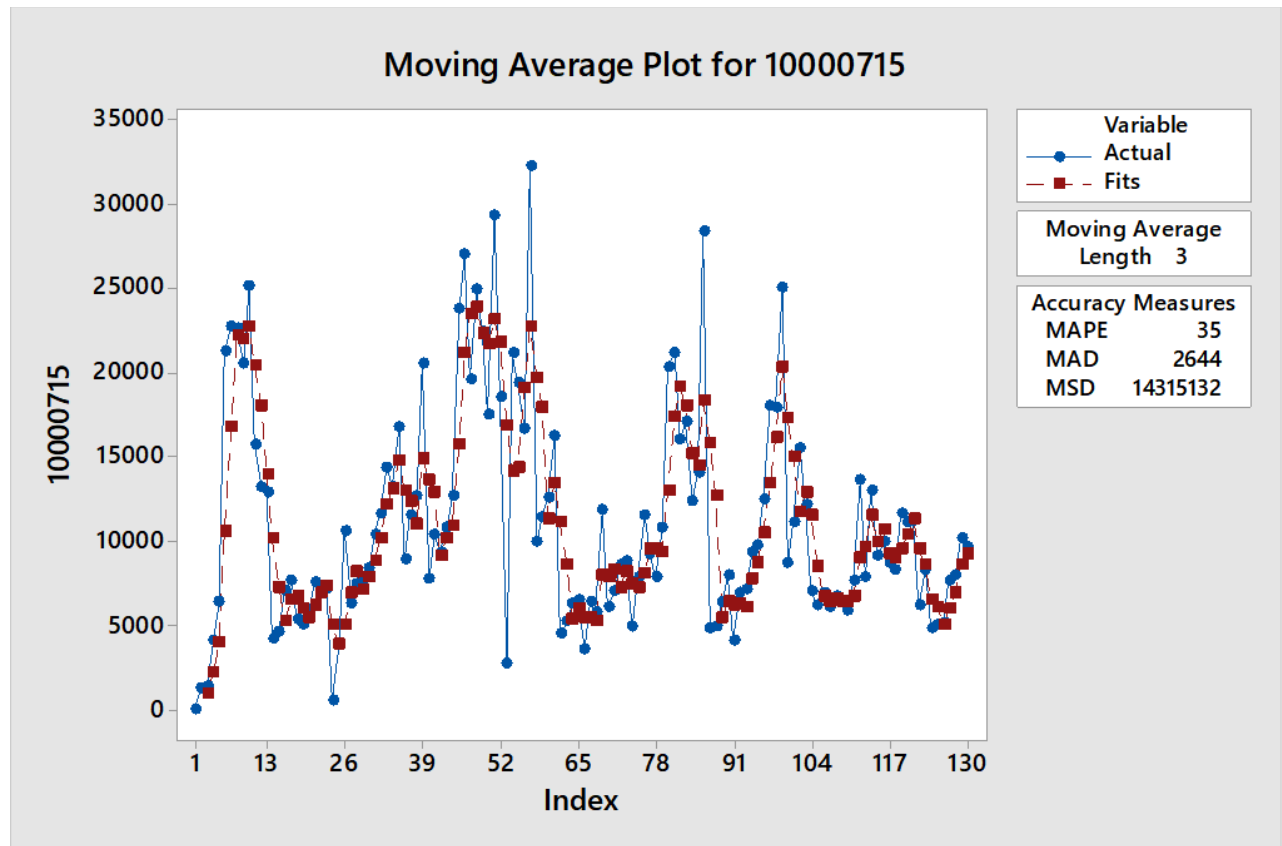

Figure 13. Moving Average SKU 10000715

(Source: Primary Data Processing, 2020)

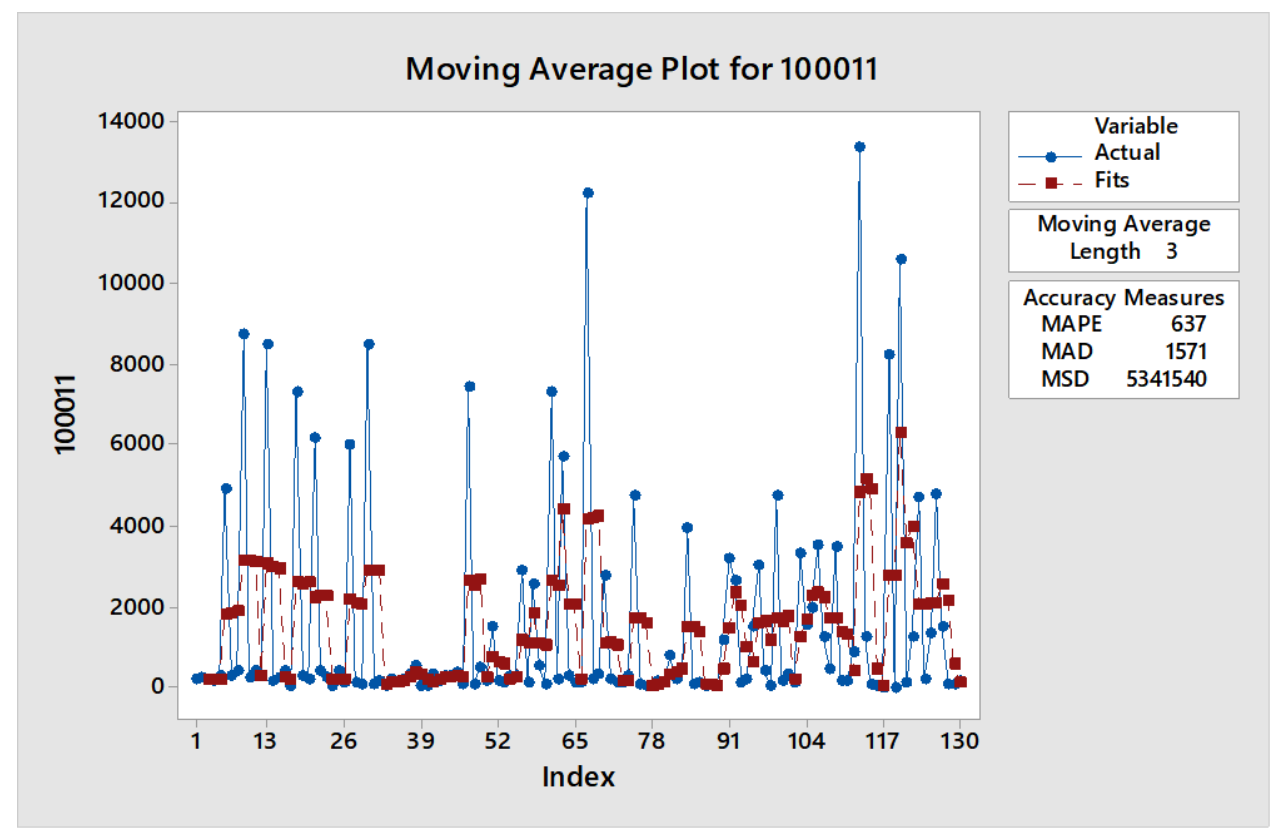

Figure 14. Moving Average SKU 100011

(Source: Primary Data Processing, 2020)

The accuracy values of the MA method are represented by MAPE, MAD, and MSE. The smaller MAPE, MAD, and MSE values indicate that the forecasting method being carried out is more accurate.

\subsection{Double Exponential Smoothing Forecasting (DES) \\ The alpha (level) and the gamma (trend) values from DES forecasting are determined}

based on the equivalence with the optimal ARIMA model. Table 2 shows the alpha and gamma values for each SKU. 
Table 2. Alpha and Gamma Values for every SKU

\begin{tabular}{ccc}
\hline SKU & Alpha & Gamma \\
\hline 10000531 & 1.0237 & 0.01207 \\
10000709 & 0.991529 & 0.002686 \\
10000736 & 1.01084 & 0.0122 \\
10000534 & 1.05909 & 0.00152 \\
10000712 & 0.980938 & 0.016811 \\
10000530 & 1.08004 & 0.01235 \\
071015 & 1.0529 & 0.01512 \\
10000710 & 1.0078 & 0.01523 \\
071024 & 1.08557 & 0.01166 \\
10000732 & 1.04981 & 0.01322 \\
10000715 & 0.727 & 0.002478 \\
100011 & 1.11076 & 0.00248 \\
\hline
\end{tabular}

(Source: Primary Data Processing, 2020)

\subsection{Linear Regression Forecasting (RL)}

The Simple Linear regression method is utilized for Linear Regression Forecasting. The independent and dependent variables are the period and number of demand from consumers, respectively. Table 3 describes the forecasting equations obtained by the linear regression method.

Table 3. Linear Regression for Every SKU

\begin{tabular}{cl}
\hline SKU & \multicolumn{1}{c}{ Equation } \\
\hline 10000531 & $Y_{t}=118561-611 \times t$ \\
10000709 & $Y_{t}=115054-75 \times t$ \\
10000736 & $Y_{t}=78073+540 \times t$ \\
10000534 & $Y_{t}=68142-175.4 \times t$ \\
10000712 & $Y_{t}=61415-81.2 \times t$ \\
10000530 & $Y_{t}=69624-288.7 \times t$ \\
071015 & $Y_{t}=29125-17.3 \times t$ \\
10000710 & $Y_{t}=28217+52.6 \times t$ \\
071024 & $Y_{t}=28217+23.6 \times t$ \\
10000732 & $Y_{t}=34566-61.6 \times t$ \\
10000715 & $Y_{t}=12132-16.2 \times t$ \\
100011 & $Y_{t}=1206+5.03 \times t$
\end{tabular}

(Source: Primary Data Processing, 2020)

Table 4. Forecasting Results for SKU 10000531

\begin{tabular}{ccccccc}
\hline \multicolumn{6}{c}{ SKU 10000531 } \\
\hline Week & ARIMA & MA & DES & RL & Current Condition & Actual Demand \\
\hline $\mathbf{1}$ & 80,061 & 95,993 & 63820.75 & 38542.922 & 9,600 & 64,295 \\
$\mathbf{2}$ & 87,804 & 95,993 & 64646.34 & 37932.1 & 73,260 & 52,023 \\
$\mathbf{3}$ & 100,015 & 95,993 & 65471.93 & 37321.278 & 34,020 & 53,266 \\
$\mathbf{4}$ & 72,712 & 95,993 & 66297.52 & 36710.456 & 105,000 & 99,087 \\
$\mathbf{5}$ & 84,711 & 95,993 & 67123.12 & 36099.635 & 24,000 & 68,817
\end{tabular}

(Source: Primary Data Processing, 2020)

Table 5. Forecasting Results for SKU 10000709

\begin{tabular}{ccccccc}
\hline \multicolumn{7}{c}{ SKU 10000709 } \\
\hline Week & ARIMA & MA & DES & RL & Current Condition & Actual Demand \\
\hline $\mathbf{1}$ & 99,005 & 121,625 & 126720.6 & $105,187.95$ & 57,876 & 127,944 \\
$\mathbf{2}$ & 102,239 & 121,625 & 125494.7 & $105,112.64$ & 96,600 & 90,080 \\
$\mathbf{3}$ & 118,535 & 121,625 & 124268.9 & $105,037.33$ & 73,500 & 99,812 \\
$\mathbf{4}$ & 116,218 & 121,625 & 123043 & $104,962.02$ & 197,316 & 180,616 \\
$\mathbf{5}$ & 100,290 & 121,625 & 121817.2 & $104,886.71$ & 63,000 & 135,670 \\
\hline
\end{tabular}

(Source: Primary Data Processing, 2020)

Table 6. Forecasting Results for SKU 10000736

\begin{tabular}{ccccccc}
\hline & \multicolumn{5}{c}{ SKU 10000736 } \\
\hline Week & ARIMA & MA & DES & RL & Current Condition & Actual Demand \\
\hline $\mathbf{1}$ & 81,208 & 103,988 & 79,670 & 148,876 & 31,560 & 79,199 \\
$\mathbf{2}$ & 101,224 & 103,988 & 80,443 & 149,416 & 75,360 & 80,313 \\
$\mathbf{3}$ & 131,934 & 103,988 & 81,216 & 149,957 & 59,280 & 78,099 \\
$\mathbf{4}$ & 85,282 & 103,988 & 81,989 & 150,497 & 117,000 & 91,346 \\
\hline
\end{tabular}




\begin{tabular}{ccccccc}
\hline \multicolumn{7}{c}{ SKU 10000736 } \\
\hline Week & ARIMA & MA & DES & RL & Current Condition & Actual Demand \\
\hline $\mathbf{5}$ & 118,815 & 103,988 & 82,762 & 151,038 & 348,000 & 72,912
\end{tabular}

(Source: Primary Data Processing, 2020)

Table 7. Forecasting Results for SKU 10000534

\begin{tabular}{ccccccc}
\hline & \multicolumn{5}{c}{ SKU 10000534 } \\
\hline Week & ARIMA & MA & DES & RL & Current Condition & Actual Demand \\
\hline $\mathbf{1}$ & 76,743 & 98,934 & 69,725 & 45,161 & 26,340 & 71,568 \\
$\mathbf{2}$ & 60,196 & 98,934 & 71,451 & 44,986 & 31,680 & 33,710 \\
$\mathbf{3}$ & 82,326 & 98,934 & 73,177 & 44,810 & 35,430 & 39,774 \\
$\mathbf{4}$ & 80,691 & 98,934 & 74,902 & 44,635 & 37,500 & 40,884 \\
$\mathbf{5}$ & 64,675 & 98,934 & 76,628 & 44,460 & 11,250 & 38,569
\end{tabular}

(Source: Primary Data Processing, 2020)

Table 8. Forecasting Results for SKU 10000712

\begin{tabular}{ccccccc}
\hline & \multicolumn{6}{c}{ SKU 10000712 } \\
\hline Week & ARIMA & MA & DES & RL & Current Condition & Actual Demand \\
\hline $\mathbf{1}$ & 69,645 & 82,536 & 82,787 & 72,048 & 36,876 & 81,941 \\
$\mathbf{2}$ & 68,834 & 82,536 & 83,577 & 72,130 & 62,076 & 61,399 \\
$\mathbf{3}$ & 68,057 & 82,536 & 84,366 & 72,211 & 60,900 & 58,738 \\
$\mathbf{4}$ & 68,837 & 82,536 & 85,156 & 72,292 & 125,916 & 92,494 \\
$\mathbf{5}$ & 68,658 & 82,536 & 85,946 & 72,373 & 21,000 & 88,485 \\
\hline
\end{tabular}

(Source: Primary Data Processing, 2020)

Table 9. Forecasting Results for SKU 10000530

\begin{tabular}{ccccccc}
\hline & \multicolumn{5}{c}{ SKU 10000530 } \\
\hline Week & ARIMA & MA & DES & RL & Current Condition & Actual Demand \\
\hline $\mathbf{1}$ & 49,554 & 71,403 & 49,865 & 31,809 & 23,430 & 51,614 \\
$\mathbf{2}$ & 44,349 & 71,403 & 50,545 & 31,520 & 52,260 & 38,714 \\
$\mathbf{3}$ & 66,899 & 71,403 & 51,225 & 31,232 & 36,000 & 36,758 \\
$\mathbf{4}$ & 54,297 & 71,403 & 51,905 & 30,943 & 58,500 & 50,362 \\
$\mathbf{5}$ & 46,809 & 71,403 & 52,585 & 30,654 & 9,461 & 47,860 \\
\hline
\end{tabular}

(Source: Primary Data Processing, 2020)

Table 10. Forecasting Results for SKU 071015

\begin{tabular}{ccccccc}
\hline & \multicolumn{5}{c}{ SKU 071015 } \\
\hline Week & ARIMA & MA & DES & RL & Current Condition & Actual Demand \\
\hline $\mathbf{1}$ & 24,676 & 38,770 & 33,761 & 26,855 & 22,698 & 33,957 \\
$\mathbf{2}$ & 22,458 & 38,770 & 33,725 & 26,837 & 5,760 & 12,992 \\
$\mathbf{3}$ & 21,160 & 38,770 & 33,690 & 26,820 & 8,640 & 11,726 \\
$\mathbf{4}$ & 20,874 & 38,770 & 33,655 & 26,803 & 22,464 & 15,082 \\
$\mathbf{5}$ & 22,391 & 38,770 & 33,619 & 26,785 & 3,911 & 9,417 \\
\hline
\end{tabular}

(Source: Primary Data Processing, 2020)

Table 11. Forecasting Results for SKU 10000710

\begin{tabular}{ccccccc}
\hline & \multicolumn{5}{c}{ SKU 10000710 } \\
\hline Week & ARIMA & MA & DES & RL & Current Condition & Actual Demand \\
\hline $\mathbf{1}$ & 31,278 & 35,023 & 23,238 & 35,114 & 28,056 & 23,329 \\
\hline
\end{tabular}




\begin{tabular}{ccccccc}
\hline \multicolumn{7}{c}{ SKU 10000710 } \\
\hline Week & ARIMA & MA & DES & RL & Current Condition & Actual Demand \\
\hline $\mathbf{2}$ & 25,516 & 35,023 & 23,314 & 35,166 & 5,376 & 30,840 \\
$\mathbf{3}$ & 40,426 & 35,023 & 23,391 & 35,219 & 39,564 & 30,481 \\
$\mathbf{4}$ & 28,748 & 35,023 & 23,467 & 35,272 & 28,560 & 45,071 \\
$\mathbf{5}$ & 30,989 & 35,023 & 23,543 & 35,324 & 81,861 & 43,487
\end{tabular}

(Source: Primary Data Processing, 2020)

Table 12. Forecasting Results for SKU 071024

\begin{tabular}{ccccccc}
\hline & \multicolumn{5}{c}{ SKU 071024 } \\
\hline Week & ARIMA & MA & DES & RL & Current Condition & Actual Demand \\
\hline $\mathbf{1}$ & 18,457 & 40,463 & 14,553 & 20,534 & 26,274 & 18,211 \\
$\mathbf{2}$ & 18,554 & 40,463 & 14,362 & 20,557 & 28,224 & 9,952 \\
$\mathbf{3}$ & 18,588 & 40,463 & 14,170 & 20,581 & 5,184 & 7,666 \\
$\mathbf{4}$ & 18,589 & 40,463 & 13,978 & 20,604 & 4,032 & 9,332 \\
$\mathbf{5}$ & 18,579 & 40,463 & 13,786 & 20,628 & 9,216 & 8,132 \\
\hline
\end{tabular}

(Source: Primary Data Processing, 2020)

Table 13. Forecasting Results for SKU 10000732

\begin{tabular}{ccccccc}
\hline Week & ARIMA & MA & DES & RL & Current Condition & Actual Demand \\
\hline $\mathbf{1}$ & 29,847 & 26,251 & 25,271 & 26,497 & 10,752 & 24,987 \\
$\mathbf{2}$ & 31,366 & 26,251 & 25,585 & 26,436 & 26,880 & 24,633 \\
$\mathbf{3}$ & 32,066 & 26,251 & 25,899 & 26,374 & 18,942 & 19,824 \\
$\mathbf{4}$ & 32,389 & 26,251 & 26,214 & 26,313 & 23,940 & 31,218 \\
$\mathbf{5}$ & 32,538 & 26,251 & 26,528 & 26,251 & 27,300 & 32,389 \\
\hline
\end{tabular}

(Source: Primary Data Processing, 2020)

Table 14. Forecasting Results for SKU 10000715

\begin{tabular}{ccccccc}
\hline & \multicolumn{5}{c}{ SKU 10000715 } \\
\hline Week & ARIMA & MA & DES & RL & Current Condition & Actual Demand \\
\hline $\mathbf{1}$ & 9,320 & 9,297 & 9,746 & 10,015 & 25,473 & 9,647 \\
$\mathbf{2}$ & 9,613 & 9,297 & 9,957 & 9,999 & 26,880 & 17,514 \\
$\mathbf{3}$ & 9,571 & 9,297 & 10,169 & 9,983 & 28,900 & 11,902 \\
$\mathbf{4}$ & 9,512 & 9,297 & 10,380 & 9,967 & 12,390 & 19,939 \\
$\mathbf{5}$ & 9,549 & 9,297 & 10,591 & 9,951 & 35,784 & 14,162 \\
\hline
\end{tabular}

(Source: Primary Data Processing, 2020)

Table 15. Forecasting Results for SKU 100011

\begin{tabular}{ccccccc}
\hline & \multicolumn{7}{c}{ SKU 100011 } \\
\hline Week & ARIMA & MA & DES & RL & Current Condition & Actual Demand \\
\hline $\mathbf{1}$ & 3,702 & 120 & 134 & 1,864 & 678 & 4,716 \\
$\mathbf{2}$ & 306 & 120 & 93 & 1,869 & 696 & 198 \\
$\mathbf{3}$ & 326 & 120 & 53 & 1,874 & 1,536 & 1,365 \\
$\mathbf{4}$ & 2,270 & 120 & 12 & 1,879 & 4,476 & 4,788 \\
$\mathbf{5}$ & 1,618 & 120 & 28 & 1,884 & 960 & 1,517
\end{tabular}

(Source: Primary Data Processing, 2020) 
Table 16. Mean Absolute Deviation (MAD)

\begin{tabular}{lccccc}
\hline \multicolumn{1}{c}{ SKU } & ARIMA & MA & DES & RL & Current Condition \\
\hline $\mathbf{1 0 0 0 0 5 3 1}$ & 28,113 & 29,733 & 11,957 & 30,176 & 29,182 \\
$\mathbf{1 0 0 0 0 7 0 9}$ & 31,920 & 26,543 & 26,504 & 29,890 & 38,454 \\
$\mathbf{1 0 0 0 0 7 3 6}$ & 67,383 & 71,184 & 60,645 & 98,333 & 74,431 \\
$\mathbf{1 0 0 0 0 5 3 4}$ & 28,025 & 54,033 & 29,013 & 10,472 & 16,461 \\
$\mathbf{1 0 0 0 0 7 1 2}$ & 14,507 & 12,287 & 11,706 & 14,082 & 29,762 \\
$\mathbf{1 0 0 0 0 5 3 0}$ & 8,564 & 26,341 & 6,863 & 13,830 & 17,805 \\
$\mathbf{0 7 1 0 1 5}$ & 9,389 & 22,135 & 17,134 & 13,026 & 11,993 \\
$\mathbf{1 0 0 0 0 7 1 0}$ & 10,408 & 7,786 & 11,251 & 7,762 & 18,832 \\
$\mathbf{0 7 1 0 2 4}$ & 7,895 & 29,804 & 4,974 & 9,922 & 7,040 \\
$\mathbf{1 0 0 0 0 7 3 2}$ & 5,031 & 4,083 & 3,635 & 4,181 & 5,946 \\
$\mathbf{1 0 0 0 0 7 1 5}$ & 5,120 & 5,336 & 4,504 & 4,797 & 14,272 \\
$\mathbf{1 0 0 0 1 1}$ & 956 & 2,397 & 2,453 & 1,662 & 1,115 \\
\hline
\end{tabular}

(Source: Primary Data Processing, 2020)

\subsection{Forecasting Results}

Five-week demand forecasting is performed by each forecasting model produced by the aforementioned methods. Tables 4 to 15 present the predicted demands resulted from each aforementioned forecasting method, the current forecasting method employed by the company, and actual demands. Also, each table is dedicated for each class of A SKU. These tables are produced to measure the forecasting accuracy of the proposed forecasting methods which will be further described in the next section.

\subsection{Forecasting Error Calculation}

This section describes forecast error calculation that shows the accuracy of the forecasting methods. Moreover, a comparison among forecasting methods is shown in this section to show the effectiveness of the proposed forecasting methods. Two types of error measurement are utilized, i.e., MAD and MAPE (Kurniagara, 2017; Anggraeni, 2019; Irawan and Laksito, 2019; Maricar, 2019; Nurprihatin, Jayadi and Tannady, 2020). Tables 16 to 17 consecutively present the results of MAD and MAPE values for each forecasting method implemented to predict the future demands of each SKU. In addition, Figures 15 and 16 show the comparison charts for MAD and MAPE values for each forecasting method, respectively. 


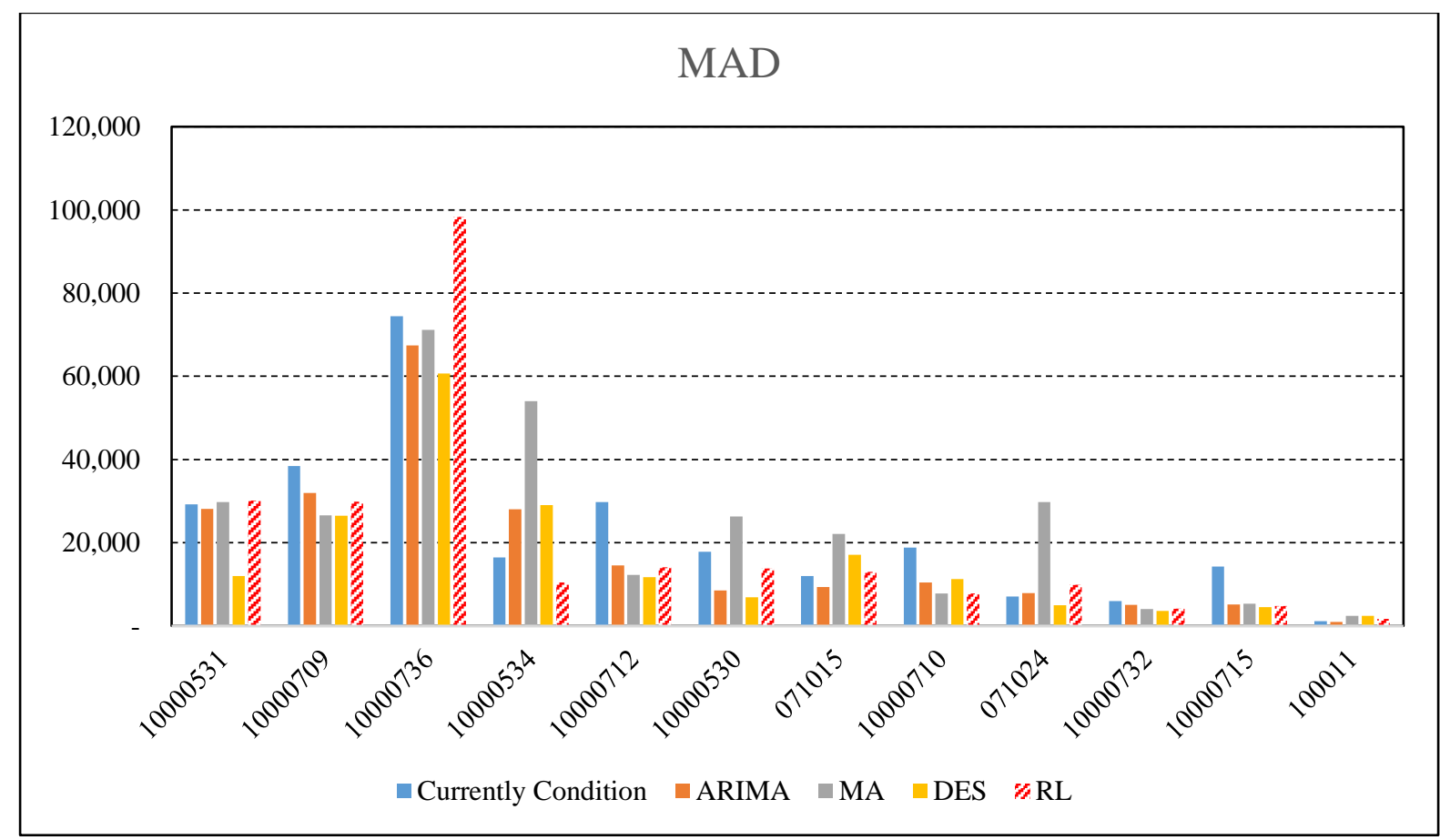

Figure 15. MAD Graph

(Source: Researcher, 2020)

Table 17. Mean Absolute Percentage Error (MAPE) Calculation

\begin{tabular}{cccccc}
\hline SKU & ARIMA & MA & DES & RL & Current Condition \\
\hline $\mathbf{1 0 0 0 0 5 3 1}$ & $32.4 \%$ & $31.0 \%$ & $18.2 \%$ & $81.4 \%$ & $169.5 \%$ \\
\hline $\mathbf{1 0 0 0 0 7 0 9}$ & $29.5 \%$ & $21.8 \%$ & $21.4 \%$ & $28.5 \%$ & $57.5 \%$ \\
\hline $\mathbf{1 0 0 0 0 7 3 6}$ & $57.0 \%$ & $68.5 \%$ & $73.3 \%$ & $65.4 \%$ & $58.0 \%$ \\
\hline $\mathbf{1 0 0 0 0 5 3 4}$ & $38.4 \%$ & $54.6 \%$ & $39.2 \%$ & $23.3 \%$ & $88.4 \%$ \\
\hline $\mathbf{1 0 0 0 0 7 1 2}$ & $21.1 \%$ & $14.9 \%$ & $13.9 \%$ & $19.5 \%$ & $94.9 \%$ \\
\hline $\mathbf{1 0 0 0 0 5 3 0}$ & $14.3 \%$ & $36.9 \%$ & $13.4 \%$ & $44.3 \%$ & $113.6 \%$ \\
\hline $\mathbf{0 7 1 0 1 5}$ & $42.0 \%$ & $57.1 \%$ & $50.9 \%$ & $48.6 \%$ & $102.9 \%$ \\
\hline $\mathbf{1 0 0 0 0 7 1 0}$ & $33.6 \%$ & $22.2 \%$ & $48.0 \%$ & $22.0 \%$ & $123.6 \%$ \\
\hline $\mathbf{0 7 1 0 2 4}$ & $42.5 \%$ & $73.7 \%$ & $35.2 \%$ & $48.2 \%$ & $57.3 \%$ \\
\hline $\mathbf{1 0 0 0 0 7 3 2}$ & $16.0 \%$ & $15.6 \%$ & $13.9 \%$ & $15.9 \%$ & $38.9 \%$ \\
\hline $\mathbf{1 0 0 0 0 7 1 5}$ & $53.6 \%$ & $57.4 \%$ & $44.0 \%$ & $48.1 \%$ & $55.4 \%$ \\
\hline $\mathbf{1 0 0 0 1 1}$ & $99.7 \%$ & $2003 \%$ & $10013 \%$ & $88.8 \%$ & $148.7 \%$ \\
\hline
\end{tabular}

(Source: Primary Data Processing, 2020) 


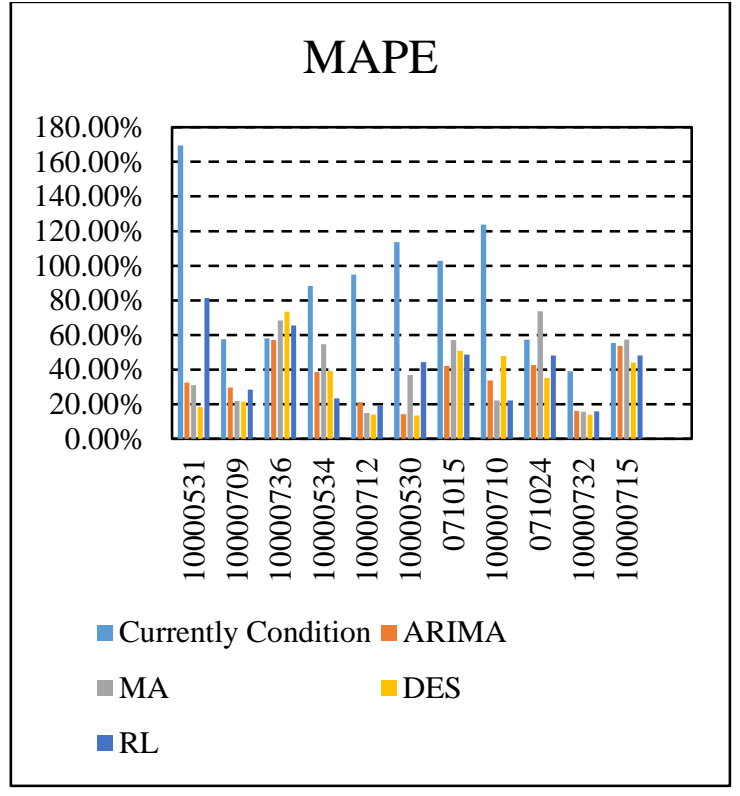

Figure 16. MAPE Graph

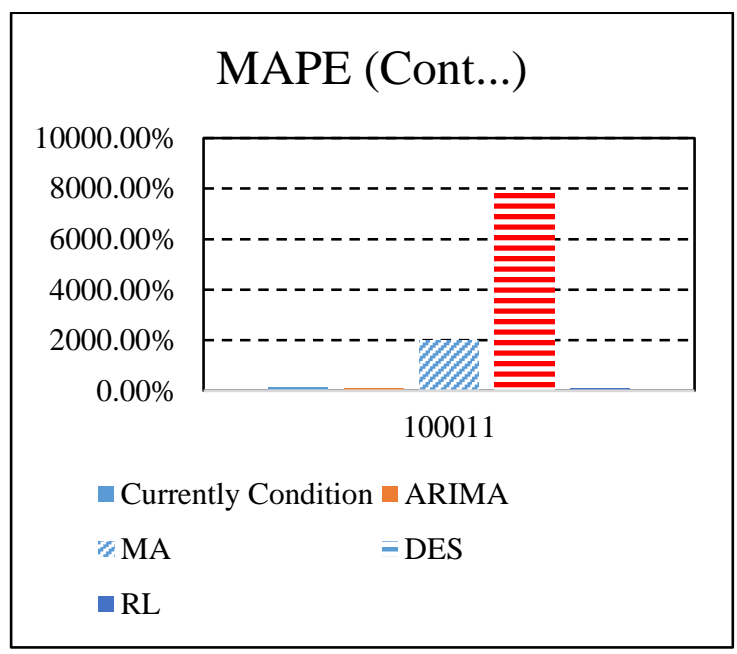

Figure 17. MAPE Graph (Cont)

(Source: Primary Data Processing, 2020)

(Source: Primary Data Processing, 2020)

Table 18. Forecasting For Every SKU

\begin{tabular}{ccc}
\hline SKU & Proposed Forecasting Method & Model \\
\hline $\mathbf{1 0 0 0 0 5 3 1}$ & DES & $1.0237,0.01207$ \\
\hline $\mathbf{1 0 0 0 0 7 0 9}$ & ARIMA & $3,0,4$ \\
\hline $\mathbf{1 0 0 0 0 7 3 6}$ & DES & $1.01084,0.01220$ \\
\hline $\mathbf{1 0 0 0 0 5 3 4}$ & Linear Regression & $Y_{t}=68142-175.4 \times t$ \\
\hline $\mathbf{1 0 0 0 0 7 1 2}$ & DES & $0.980938,0.016811$ \\
\hline $\mathbf{1 0 0 0 0 5 3 0}$ & DES & $1.08004,0.01235$ \\
\hline $\mathbf{7 1 0 1 5}$ & ARIMA & $3,0,3$ \\
\hline $\mathbf{1 0 0 0 0 7 1 0}$ & Linear Regression & $Y_{t}=28217+52.6 \times t$ \\
\hline $\mathbf{7 1 0 2 4}$ & DES & $1.08557,0.01166$ \\
\hline $\mathbf{1 0 0 0 0 7 3 2}$ & DES & $1.04981,0.01322$ \\
\hline $\mathbf{1 0 0 0 0 7 1 5}$ & DES & $0.727,0.0024778$ \\
\hline $\mathbf{1 0 0 0 1 1}$ & ARIMA & $4,1,3$ \\
\hline
\end{tabular}

(Source: Primary Data Processing, 2020)

Then determine the appropriate forecasting method for each SKU. Table 18 describes the appropriate forecasting methods to be used for each SKU.

\section{CONCLUSION}

Based on the research results, it can be concluded that the forecasting method used by the company today produces a higher error value than the time series forecasting method. Therefore, the company needs to make improvements for predicting the demands of each SKU.
The results show that each SKU requires a different forecasting method, depending on the historical data characteristics of each SKU. Thus, the implementation of various forecasting methods is intended to obtain forecasting results with higher accuracy. The most appropriate forecasting methods used for forecasting each SKU are listed as follows: (1) double exponential smoothing for SKU 10000531, SKU 10000736, SKU 10000712, SKU 071024, SKU 10000732, SKU 10000715 (2) ARIMA for SKU 10000709, SKU 10000530, SKU 071015, SKU 100011 (3) linear regression for SKU 10000534, SKU 10000710. Based on these 
results, double exponential smoothing is the most suitable forecasting method among all the employed forecasting methods.

The suggestions related to future research are to perform classification on SKU classes to ensure the SKU availability and inventory in the warehouse management system, to control orders and store goods by considering demand uncertainty and waiting time, and to calculate cycle service levels (CSL) by controlling distribution logistics. Further study can also discuss 8 pillars of TPM and 5S to increase the effectiveness (Nurprihatin, Angely and Tannady, 2019).

\section{REFERENCES}

Anggraeni, D. T. (2019) "Forecasting Harga Saham Menggunakan Metode Simple Moving Average dan Web Scrapping," Jurnal Ilmiah Matrik, 21(3), pp. 234-241.

Chopra, S. and Meindl, P. (2016) Supply Chain Management: Strategy, Planning, and Operation. 6th ed. New York: Pearson Education.

Christian, M. et al. (2021) "Business Performance Determinants of Salted Fish Distribution in Kapuk During the COVID-19," Journal of Distribution Science, 19(6), pp. 29-39. doi: 10.15722/jds.19.6.202106.29.

Hirschmann, R. (2020) Contribution to GDP by Industry Indonesia 2019, Statista.

Irawan, R. Y. and Laksito, W. (2019) "Penerapan Metode Double Exponential Smoothing Untuk Peramalan Tingkat Indeks Pembangunan Manusia," Jurnal TIKomSiN, 7(2), pp. 18-28.

Kurniagara (2017) "Penerapan Metode Exponential Smoothing Dalam Memprediksi Jumlah Siswa Baru (Studi Kasus: SMK Pemuda Lubuk Pakam)," Jurnal Pelita Informatika, 6, pp. 19-25.

Maricar, M. A. (2019) "Analisa Perbandingan Nilai Akurasi Moving Average dan Exponential Smoothing untuk Sistem Peramalan Pendapatan pada Perusahaan XYZ," Jurnal Sistem dan Informatika, 13(2), pp. 36-45.

Nurprihatin, F. et al. (2019) "A Distribution
Strategy Using A Two-step Optimization to Maximize Blood Services Considering Stochastic Travel Times," in IOP Conference Series: Materials Science and Engineering. doi: 10.1088/1757899X/650/1/012043.

Nurprihatin, Filscha et al. (2019) "The Extension Analysis of Natural Gas Network Location-Routing Design Through the Feasibility Study," Journal of Applied Research on Industrial Engineering, 6(2), pp. 108-124. doi: 10.22105/jarie.2019.174164.1082.

Nurprihatin, F., Angely, M. and Tannady, H. (2019) "Total Productive Maintenance Policy to Increase Effectiveness and Maintenance Performance Using Overall Equipment Effectiveness," Journal of Applied Research on Industrial Engineering, 6(3), pp. 184-199. doi: 10.22105/jarie.2019.199037.1104.

Nurprihatin, F., Jayadi, E. L. and Tannady, H. (2020) "Comparing Heuristic Methods' Performance for Pure Flow Shop Scheduling Under Certain and Uncertain Demand," Management and Production Engineering Review, 11(2), pp. 50-61. doi: 10.24425/mper.2020.133728.

Nurprihatin, F. and Lestari, A. (2020) "Waste Collection Vehicle Routing Problem Model with Multiple Trips, Time Windows, Split Delivery, Heterogeneous Fleet and Intermediate Facility," Engineering Journal, 24(5). doi: 10.4186/ej.2020.24.5.55.

Tannady, H. et al. (2019) "Process Improvement to Reduce Waste in the Biggest Instant Noodle Manufacturing Company," Journal of Applied Engineering Science, 17(2), pp. 203-212. doi: 10.5937/jaes1718951.

Tannady, H., Nurprihatin, F. and Hartono, H. (2018) "Service Quality Analysis of Two of the Largest Retail Chains with Minimart Concept in Indonesia," Business: Theory and Practice, 19, pp. 177-185. doi: 10.3846/BTP.2018.18. 